Relations industrielles

Industrial Relations

\title{
Wheeler, H.N., and J. Rojot, eds. Workplace Justice. Employment Obligations in International Perspective
}

\section{Pierre Verge}

Volume 48, numéro 3, 1993

URI : https://id.erudit.org/iderudit/050884ar

DOI : https://doi.org/10.7202/050884ar

Aller au sommaire du numéro

Éditeur(s)

Département des relations industrielles de l'Université Laval

ISSN

0034-379X (imprimé)

1703-8138 (numérique)

Découvrir la revue

Citer ce compte rendu

Verge, P. (1993). Compte rendu de [Wheeler, H.N., and J. Rojot, eds. Workplace Justice. Employment Obligations in International Perspective]. Relations industrielles / Industrial Relations, 48(3), 584-585.

https://doi.org/10.7202/050884ar

Tous droits réservés (C) Département des relations industrielles de l'Université Laval, 1993
Ce document est protégé par la loi sur le droit d'auteur. L'utilisation des services d'Érudit (y compris la reproduction) est assujettie à sa politique d'utilisation que vous pouvez consulter en ligne.

https://apropos.erudit.org/fr/usagers/politique-dutilisation/ 
que Fernand Morin récidive sous peu en nous offrant un autre ouvrage en droit du travail car il est, au Québec, un auteur phare.

Jean-Yyes BrikRE

Montréal

Workplace Justice - Employment Obligations in International Perspective, by H.N. Wheeler and J. Rojot, eds., Columbia, University of South Carolina Press, 1992, 387 p., ISBN 0-87249-781-X

Les premières lignes de l'ouvrage évoquent la scène fictive d'un préposé à l'entretien qui refuse avec véhémence d'obtempérer à l'intimation de son contremaître de laver à nouveau un plancher : comment les institutions du travail et le droit traiteront-ils cette situation? Ces lignes illustrent, en fait, l'originalité, comme nous le verrons, de cette entreprise collective, sous la direction des professeurs Hoyt $N$. Wheeler de University of South Carolina et de Jacques Rojot, de Paris I, visant à comparer le droit applicable aux situations disciplinaires-droit commun et droit du travail, selon leur pertinence respective - dans certains pays: Australie, Belgique, Canada, France, Allemagne, Israël, Italie, Espagne, Royaume-Uni et États-Unis. Plus exactement, comme le veut d'ailleurs l'ensemble du titre, il s'agit d'abord de faire ressortir dans chaque cas le contenu obligationnel essentiel du statut de salarié pour ensuite suivre dans son prolongement procédural, au sens large, la situation disciplinaire, qu'elle ait donné lieu au renvoi du salarié, ou à une sanction moindre. Il s'agira, en finale, de rapprocher ces différents portraits nationaux, de voir, en définitive, si des données communes ne les sous-tendent pas.

D'une facon plutôt innovatrice - et heureuse doit-on convenir - dans le genre, les rapporteurs nationaux ont accepté de respecter une grille factuelle d'analyse : quel serait dans leur propre juridiction le traitement de dix scénarios disciplinaires concrets? Ceux-ci évoquent tour à tour l'insubordination, la consommation d'alcool au travail, la subtilisation d'un bien de l'entreprise, une altercation entre salariés, l'absentéisme, l'insuffisance du rendement, une atteinte à la réputation de l'entreprise de la part du salarié, son incitation à faire une grève sur le tas et, finalement, le travail d'appoint pour une entreprise concurrente. Dans chaque cas, les rapporteurs eurent à tenir compte de différents facteurs, également prescrits, atténuants aussi bien qu'aggravants.

Une synthèse du droit national applicable précède avec bonheur ces analyses. Ainsi, dans le cas du Canada, les professeurs Roy J. Adams et Bernard Adell font état de la dualité de régime du travail, selon qu'il est, ou non, régi par une convention collective. L'affirmative conduit à un exposé succinct, mais néanmoins précis et essentiel, des principes directeurs qui se dégagent de la jurisprudence arbitrale relative au contrôle de la fonction disciplinaire dans l'entreprise; la négative entraîne les solutions connues du droit commun, de la common law devrait-on dire plus restrictivement, car les auteurs s'abstiennent de toute allusion aux solutions civilistes qui ont cours dans une partie du pays. Lacune regrettable, malgré la similitude d'ensemble des solutions à ce niveau convenu de généralisation, car l'entreprise, en était une au départ de droit comparé... (ll est 
seulement fait mention de l'existence au Québec, en Nouvelle-Écosse et dans l'ordre fédéral de l'arbitrage légal relatif au congédiement injuste.)

Ces courtes synthèses diffèrent quelque peu formellement entre elles. Ainsi, le texte américain, qui doit également faire état d'une semblable dualité de régime, insiste sur le développement historique de dérogations législatives et jurisprudentielles au employment at will. On y trouve également une comparaison de la notion traditionnelle de « cause juste » - issue du contrat a durée déterminée - et de son acception moderne. La synthèse allemande, toujours à titre illustratif, ne peut manquer de faire état des rôles respectifs de la négociation collective et des institutions de codétermination; elle traite également des tribunaux du travail et insiste sur les modes de sanction des obligations du salarié. Le rapport italien présente de façon liminaire la conception du pouvoir disciplinaire entretenue par le droit de ce pays au fil de son histoire et met en relief l'importance du rôle actuel de la convention collective. Le professeur Rojot, non seulement présente-t-il, malgré sa relative complexité, les aspects saillants du système français et des lois, notamment de 1973 et de 1982, qui l'ont façonné, mais s'interroge également sur le fondement, institutionnel ou contractuel, du pouvoir de direction de l'employeur, réflexion dont on aimerait connaître le pendant, dans la mesure où il peut exister, dans d'autres pays.

Tout cela cependant risque de distraire de ce qui, encore une fois, constitue la dimension caractéristique de la démarche : les analyses situationnelles concrètes. Leur résultat est évidemment tributaire des paramètres juridiques applicables. Or, ceux-ci diffèrent fondamentalement à l'occasion, par exemple relativement à l'incitation à la grève: liberté fondamentale, non généralement réglementée (France, Italie); illégalité, au contraire, d'un tel comportement en cours de convention collective : Canada (obligation légale de paix), États-Unis (obligation conventionnelle); Allemagne ... Malgré tout, la conclusion générale, tout en signalant des disparités d'intensité de différents devoirs se rattachant au statut de salarié, retient néanmoins un dénominateur commun significatif à travers les différentes manifestations nationales de ce statut. Ne procède-t-il pas toujours, quelle que soit la nature du système juridique en cause, d'une situation de subordination?

Pierre Verge

Université Laval

Les normes du travail, $2^{\circ}$ éd., par Jean-Louis Dubé et Nicola D'lorio, Les Éditions Revue de Droit, Université de Sherbrooke, 1992, 779 p., ISBN 2-920003-17-8

Cinq ans après la parution de la première édition de cet ouvrage, ses mêmes auteurs nous offrent une autre version qui se veut une mise à jour, notamment pour prendre en compte les modifications apportées à la Loi sur les normes du travail en 1990 (L.Q. 1990, c. 73) et l'évolution de la jurisprudence. En raison de nombreux points communs entre ces deux publications (le même énoncé principal de la loi, le même plan et les mêmes auteurs), il y a lieu de référer le lecteur à notre recension initiale $(1987,42$ Relations industrielles 655) pour éviter quelques redites ou doublons. 